\title{
Scattering Resonances of a Cylindrical Plasma
}

\author{
William M. Leavens
}

Central Radio Propagation Laboratory, National Bureau of Standards, Boulder, Colo.

(Received May 26, 1965)

\begin{abstract}
The Vlasov equation has been solved for the plasma resonance spectra of a realistic model by the conductivity kernel method. The results give a clearer picture of the nature of plasma resonance than heretofore available. The calculation includes Landau damping and does not impose unphysical boundary conditions. The problem of chief interest here is the scattering of electromagnetic radiation near the electron plasma frequency from a cylindrical plasma when the wave vector and polarization are perpendicular to the cylinder axis. The plasma is nonuniform and bounded by a sheath, and has diameter $a$ small compared to the free space wavelength. The scattering resonances at the lower frequencies are produced by charge density perturbations concentrated at relatively large radii. But, the problem of a perfectly collisionless cylindrical plasma cannot be reduced to a one-dimensional problem without neglecting some of the resonances with periodic electron orbits. It is argued that weak coulomb collisions destroy these "transit time resonances," and that the problem is adequately described by keeping just one period of the electron orbit in the calculation of the conductivity kernel. The cylindrical problem then reduces to the problem of the steady, driven, oscillations of a thin onedimensional slab of collisionless, Maxwellian, plasma, with a wall at $x=0$ which emits electrons and absorbs all electrons that return to $x=0$. and an insulated wall at $x=x_{w}$ which also absorbs electrons. A model is used in which the unperturbed electric field, everywhere in the positive $x$-direction, is uniform in the plasma, $0 \leqslant \times \leqslant s$, and joins smoothly to a harmonic oscillator field in the sheath, $s \leqslant \mathrm{X} \leqslant x_{w}$. The conductivity kernels for a large number of frequencies have been calculated, and inverted, on large electronic computers. The results show that much of the Landau damping which determines the line shapes is concentrated near the sheath, and the resonance frequencies are determined by the properties of the sheath and the neighboring regions of the plasma.
\end{abstract}

\section{Introduction}

"Plasma resonance" is the name given by Tonks [1931a, b] to the resonances he found near the plasma frequency in a system consisting of the cylindrical positive column of a low-pressure mercury arc placed between a pair of capacitor plates which were driven by an oscillator, when the plasma axis was perpendicular to the electric field. Plasma resonance has since been found in free-space scattering of microwaves from the positive column [Romell, 1951], in backscatter of 55 megacycle radiation from meteor trails [Billam and Browne, 1955], in the reflection from the positive column placed across a waveguide [Dattner, 1957], and in noise radiation [Lustig, 1964]. Our purpose in this paper is to obtain a reasonable theoretical understanding of plasma resonance in the absence of a magnetic field. We calculate the response of a nearly collisionless plasma to an externally applied weak driving field for frequencies on the order of the mean plasma frequency. We include the nonuniformity of the plasma and the sheath and do not introduce arbitrary boundary conditions.

Tonks attempted to explain the strongest resonance as follows: Consider a sharply bounded cylinder of cold, neutral, uniform plasma in free space. Let $N$ be the electron density, $m$ the electron mass, and $e$

${ }^{1}$ Most of this work was done at the University of California at San Diego, and was in cluded in the author's Ph.D. thesis. the magnitude of the electron charge. A slight uniform displacement, $\delta \chi$, of the electrons with respect to the heavy and therefore nearly stationary ions produces a surface charge density $-N e \delta \chi \cos \theta$, and a uniform restoring force inside the plasma of $-2 \pi$ $N e^{2} \delta \chi$. The system should oscillate at the angular frequency $\omega$, with $\omega=\omega_{p} / \sqrt{2}$, where $\omega_{p}=\left(4 \pi N e^{2} / m\right)^{1 / 2}$ is the plasma frequency, if the internal motion of the surface charge is ignored. Tonks identified his strongest resonance with the response of this dipole plasma mode to the driving field. The observed angular frequency was near $\omega_{p} / \sqrt{2}$.

An equivalent model, an infinitely long cylinder, which was thin compared to $\lambda=2 \pi \mathrm{c} / \omega$, and had dielectric constant $1-\omega_{p}^{2} / \omega^{2}$, was used to predict the backscatter from meteor trails [Herlofson, 1951]. The experimental results for meteor trails [Billam and Browne, 1955] are quite interesting. The authors were apparently only interested in the main peak, but their results seem to indicate that there may be as many as six resonances, while the theory predicted one. Tonks [1931a, b] suggested that the one or two "anomalous" resonances he observed were caused by the nonuniform electron density and nonzero plasma temperature. Theoretical work since Herlofson's paper [1951] has been directed toward taking adequate account of these two conditions. The theory [Herlofson, 1951] also predicted that the scattering width at resonance would be $8 \lambda / \pi$, where the scattering width 


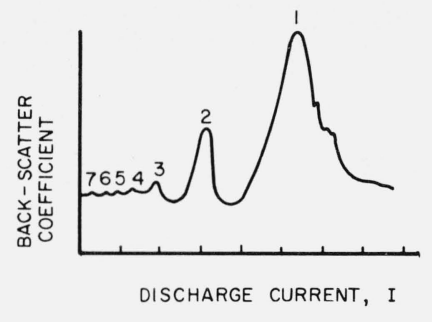

FIGURE 1. Microwave reflection from a positive column placed across a waveguide; taken from Dattner [1957].

was defined as the diameter of a cylinder which would absorb and then reradiate isotropically all of the radiation incident on it, and produce the same backscattered intensity as the actual scatterer. Romell [1951] found that at $\lambda=30 \mathrm{~cm}$, a $3.2 \mathrm{~cm}$ diameter cylindrical discharge plasma gave backscatter intensity at the first resonance which was 0.8 that of a long flat copper strip which was $15 \mathrm{~cm}$ wide. At least the first two resonances excited by plane waves are essentially pure dipole modes [Boley, 1958], as required by the theory. Higher multipoles have been excited by other driving field configurations [Parker, Nickel, and Gould, 1964].

Dattner [1957, 1963] has experimented with the cylindrical positive column of a mercury arc placed across a waveguide with its axis perpendicular to the $E$-vector. He has demonstrated that there are often six or seven resonances (fig. 1). The strongest falls at the highest arc current (lowest $\omega / \omega_{p}$ ), and they form a regular sequence. Dattner also introduced the concept of the "series limit," i.e., a definite upper limit on the resonance frequencies.

The steady state structure of the positive column and sheath is only beginning to become well understood [Ott, 1963; Self, 1963]. Where characteristics of the steady arc are needed we shall use experimental data [Gabor, Ash, and Dracott. 1955; Gierke, Ott, and Schwirzke, 1961; Harp and Kino, 1963].

The kinetic theory of plasma resonance is developed, in section 2, by the conductivity kernel method. For comparison with the kinetic theory, the adiabatic fluid equations are solved in section 3 . The fluid theory is unsatisfactory for calculating the electric fields. Nevertheless, with a carefully chosen boundary condition, it is capable of predicting the resonance frequencies.

\section{Kinetic Theory of Plasma Resonance}

Drummond, Gerwin, and Springer [1961] have derived a general integrodifferential equation for weak, oscillating electric fields in a collisionless plasma. We give a simple derivation of the conductivity kernel equation, for cases where the perturbed magnetic field may be neglected, in section 1. Sections 2-2.5, describe the simplifications which lead from the cylinder problem to the one-dimensional problem that we have solved, and sections 2.6 and 2.7 are descriptions of the numerical solution and the results.

\subsection{Conductivity Kernel Equation}

Maxwell's equations,

$$
\begin{gathered}
\boldsymbol{\nabla} \cdot \mathbf{B}=0, \\
c \boldsymbol{\nabla} \times \mathbf{B}=4 \pi \mathbf{j}+\partial \mathbf{E} / \partial t,
\end{gathered}
$$

and

$$
c \boldsymbol{\nabla} \times \mathbf{E}=-\partial \mathbf{B} / \partial t,
$$

with the usual symbols, (Gaussian units except $j$, in esu; i.e., $j=\mathrm{Nev}$ ), imply the general wave equation

$$
\boldsymbol{\nabla}{ }^{2} \mathbf{E}-\frac{1}{c^{2}} \frac{\partial^{2} \mathbf{E}}{\partial t^{2}}=\frac{4 \pi}{c^{2}} \frac{\partial \mathbf{j}}{\partial t}+4 \pi \boldsymbol{\nabla} \rho .
$$

Conductors and dielectrics external to the plasma could be included by using the constitutive equations [Panofsky and Phillips, 1962], but have no fundamental role in plasma resonance.

The source terms are related to the distribution function $F_{s}$ of species $s$ (where $s= \pm$ stands for singly charged ions or electrons respectively) by

$$
\begin{aligned}
& \mathbf{j}=e \sum_{s} s \int_{-\infty}^{\infty} \mathbf{v} F_{s}(\mathbf{v}) d^{3} v, \\
& \rho=e \sum_{s} s \int_{-\infty}^{\infty} F_{s}(\mathbf{v}) d^{3} v .
\end{aligned}
$$

The identity

$$
F_{s}(\mathbf{x}, \mathbf{v}, t)=F_{s}\left(\mathbf{x}_{0}, \mathbf{v}_{0}, t_{0}\right)+\int_{t_{0}}^{t} \frac{d F_{s}\left(\mathbf{x}^{\prime}, \mathbf{v}^{\prime}, t^{\prime}\right)}{d t^{\prime}} d t^{\prime},
$$

where the set of points $\left(\mathbf{x}^{\prime}\left(\mathbf{x}, \mathbf{v}, t^{\prime}\right), \mathbf{v}^{\prime}\left(\mathbf{x}, \mathbf{v}, t^{\prime}\right)\right)$ lie on any continuous path $S$ through $(\mathbf{x}, \mathbf{v})$, provides the connection with the kinetic equation,

$$
\frac{d F_{s}}{d t}=\frac{\partial F_{s}}{\partial t} \mid \text { collisions }
$$

where $S$ must now be the mean path in phase space of the particles in a small neighborhood around $\left(\mathbf{x}^{\prime}, \mathbf{v}^{\prime}\right)$.

Consider small, oscillatory perturbations about the steady state,

$$
F_{s}=N_{0 s} f_{0 s}+f_{s} e^{-i \omega t},
$$

where

$$
\int_{-\infty}^{\infty} f_{0 s}(\mathbf{x}, \mathbf{v}) d^{3} v=1,
$$

and

$$
A=A_{0}+A_{1} e^{-i \omega t},
$$


where $A=\mathbf{E}, \mathbf{B}, \mathbf{j}$, or $\rho$, and where $N_{0 s}(\mathbf{x})$ is the unperturbed density, and where $\omega$ is of the order of the electron plasma frequency.

In the discharges where resonance has been observed, the frequency of momentum change by collisions is very small compared to the plasma frequency so we tentatively write the linearized Vlasov equation for $f_{s}$,

$$
\frac{d f_{s}}{d t}=-\frac{s e}{m_{s}} \mathbf{E}_{1} \cdot \boldsymbol{\nabla}_{v} f_{0 s}(\mathbf{x}, \mathbf{v}) N_{0 s}(\mathbf{x})
$$

Collisions acutally play an important role, which is described in section 2.7. We have neglected the $\mathbf{v} \times \mathbf{B}_{1} / c$ term on the right-hand side of (7), which is valid for high frequency modes or for isotropic $f_{0 s}$.

The zero order part of the set of equations (2) to (5) is complicated by the collision terms. We assume solutions of this set, and use experimental data[Gabor, Ash, and Dracott, 1955; Gierke, Ott, and Schwirzke, 1961; Harp and Kino, 1963] when numbers are needed. This process is consistent only if no high frequency phenomena play an essential part in determining the measured "steady-state" [Gabor, Ash, and Dracott, 1955; Ott, 1963].

Substituting eq (7) into (4), eq (4) into (3) and eq (3) into (2), we obtain the following linearized equation for $\mathbf{E}_{1}(\mathbf{x})$ :

$$
\begin{aligned}
\nabla^{2} \mathbf{E}_{1}+\frac{\omega^{2}}{c^{2}} \mathbf{E}_{1}=-e^{i \omega t} \sum_{s} \int_{-\infty}^{\infty}\left(\boldsymbol{\nabla}-\frac{i \omega}{c^{2}} \mathbf{v}\right) \\
\int_{-\infty}^{t} \mathbf{E}_{1} \cdot \boldsymbol{\nabla} v^{\prime} f_{0 s}\left(\mathbf{x}^{\prime}, \mathbf{v}^{\prime}\right) e^{-i \omega t^{\prime}} \omega_{s}^{2}\left(\mathbf{x}^{\prime}\right) d t^{\prime} d^{3} v,
\end{aligned}
$$

where $\omega_{s}^{2}=4 \pi N_{0 s} e^{2} / m_{s}$. The quantities $\mathbf{x}^{\prime}\left(\mathbf{x}, \mathbf{v}, t^{\prime}\right)$, $\mathbf{v}^{\prime}\left(\mathbf{x}, \mathbf{v}, t^{\prime}\right)$ are the position and velocity at time $t^{\prime}$ on the orbit that goes through $(\mathbf{x}, \mathbf{v})$ at $t^{\prime}=t$. Equation (8) assumes the initial conditions

$$
f_{s}\left(\mathbf{x}, \mathbf{v}, t_{0} \rightarrow-\infty\right)=0,
$$

so we must follow the Landau convention [Landau, 1946; Thompson, 1962] with respect to any poles in the integrals in (8). Let $x_{i}(\mathbf{v})$ be a particular direction such that $v_{i}^{\prime} \neq 0$ except at isolated turning points, and write $d t^{\prime}$ as $d x_{i}^{\prime} / v_{i}^{\prime}$. Let $p$ be the number of times (counting backward in time from $t$ ) that the orbit through $(\mathbf{x}, \mathbf{v}, t)$ has passed through a particular $x_{i}^{\prime}$. The path integral along each passage of the orbit is expressed as a space integral by writing the integral as a set of delta functions located on the orbit, i.e., let

$$
\underline{e}_{i}^{-i \omega\left(t^{\prime}-t\right)}=e^{-i \omega\left(t^{\prime}-t\right)} u\left(x_{i}^{\prime}-x_{i, t p}\right) \delta\left(\mathbf{x}_{\perp}^{\prime}\right),
$$

where $\mathbf{x}_{\perp}^{\prime}$ is in the plane perpendicular to $x_{i}^{\prime}$ at the position $\mathbf{x}^{\prime}$ on the orbit, $x_{i . t p}$ is the turning point, and $u\left(x_{i}^{\prime}-x_{i, t p}\right)$ is a step function such that the amplitude is zero beyond the turning point, and where we must take $e^{-i \omega\left(t^{\prime}-t\right)}$ to be nonsingular at the off-orbit points where it is undefined. Also define

$$
\sigma_{p i}=v_{i}^{\prime}\left(\mathbf{x}, \mathbf{v} ; \mathbf{x}^{\prime}, p\right) / v_{i}^{\prime}\left(\mathbf{x}, \mathbf{v} ; \mathbf{x}^{\prime}, 1\right),
$$

which is either of \pm 1 . Now write the orbit integral as a space integral

$$
\begin{aligned}
& \int_{-\infty}^{t} \mathbf{E}_{1}\left(\mathbf{x}^{\prime}\right) \cdot \boldsymbol{\nabla} v^{\prime} f_{0 s}\left(\mathbf{v}^{\prime}\right) e^{-i \omega t^{\prime}} \omega_{s}^{2}\left(\mathbf{x}^{\prime}\right) d t^{\prime} \\
& =\sum_{p} \sigma_{p i} \int \mathbf{E}_{1}\left(\mathbf{x}^{\prime}\right) \cdot \boldsymbol{\nabla} v^{\prime} f_{0 s}\left(\mathbf{v}^{\prime}\right) \underline{e_{i}^{-i \omega t^{\prime}}} \omega_{s}^{2}\left(\mathbf{x}^{\prime}\right) \frac{1}{v_{i}^{\prime}} d^{3} x^{\prime},
\end{aligned}
$$

where the integral extends over all regions which are reached by any electrons, i.e., the limits of the space integration are independent of velocity.

Using (12) in (8) and exchanging the order of integration gives

$$
\nabla^{2} \mathbf{E}_{1}+\frac{\omega^{2}}{c^{2}} \mathbf{E}_{1}=\int \mathscr{T}\left(\mathbf{x} ; \mathbf{x}^{\prime}\right) \cdot \mathbf{E}_{1}\left(\mathbf{x}^{\prime}\right) d^{3} x^{\prime},
$$

where

$$
\begin{aligned}
\mathscr{T}\left(\mathbf{x} ; \mathbf{x}^{\prime}\right)= & \sum_{s, p} \int_{-\infty}^{\infty} \\
& \sigma_{p i}\left(\boldsymbol{\nabla}-\frac{i \omega}{c^{2}} \mathbf{v}\right) \underline{e}_{i}^{-i \omega\left(t^{\prime}-t\right)} \omega_{s}^{2}\left(\mathbf{x}^{\prime}\right) \boldsymbol{\nabla}_{v^{\prime}} f_{0 s} \frac{d^{3} v}{v_{i}^{\prime}} .
\end{aligned}
$$

Equation (13) is the conductivity kernel equation [Drummond, Gerwin, and Springer, 1961].

If the plasma thickness, $a$, in the direction of the dominant fields is very small, i.e., $k a<<1$, where $k=\omega / c$, then we may describe the processes inside the plasma by the static approximation, $\boldsymbol{\nabla} \times \mathbf{E}_{1}$ $=0, \mathbf{B}_{1}=0$, most easily obtained by setting $l / c=0$ in Maxwell's equations. Then, since

$$
\boldsymbol{\nabla} \times \boldsymbol{\nabla} \times \mathbf{E}_{1}=0,
$$

we have

$$
\nabla^{2} \mathbf{E}_{1}=\boldsymbol{\nabla} \boldsymbol{\nabla} \cdot \mathbf{E}_{1},
$$

which, when combined with Poisson's equation, indicates that the wave equation, (2), splits into two separate equations,

$$
\nabla^{2} \mathbf{E}_{1}=4 \pi \boldsymbol{\nabla} \rho_{1},
$$

and

$$
\partial \mathbf{E}_{1} / \partial t=-4 \pi \mathbf{j}_{1}
$$

Equation (13) splits similarly, the more useful equation being

$$
\mathbf{E}_{1}(\mathbf{x})=\int \mathscr{S}\left(\mathbf{x} ; \mathbf{x}^{\prime}\right) \cdot E_{1}\left(\mathbf{x}^{\prime}\right) d^{3} x^{\prime},
$$


where

$$
\mathscr{S}\left(\mathbf{x} ; \mathbf{x}^{\prime}\right)=\frac{-i}{\omega} \sum_{s, p} \int_{-\infty}^{\infty} \sigma_{p i} \frac{\mathbf{v}}{v_{i}^{\prime}} \underline{e}_{i}^{-i \omega\left(t^{\prime}-t\right)} \omega_{s}^{2}\left(\mathbf{x}^{\prime}\right) \boldsymbol{\nabla}_{v_{s}^{\prime}} f_{0 s} d^{3} v .
$$

If $\mathbf{E}_{1}=\mathbf{E}_{p}+\mathbf{E}_{d}$, where $\mathbf{E}_{d}$ is a driving field with sources far outside the plasma, only $\mathbf{E}_{p}$ can satisfy the electrostatic approximation, and since $\mathbf{E}_{d}$ satisfies the free space wave equation, (13') becomes

$$
\mathbf{E}_{p}=\int \mathscr{S} \cdot\left(\mathbf{E}_{p}+\mathbf{E}_{d}\right) d^{3} x^{\prime}
$$

The electrostatic approximation is valid whenever the characteristic length is short compared to the free-space wavelength.

\subsection{Positive Column, $\omega \approx \omega_{-}$}

In the positive column the electron distribution function $f_{0-}$, is observed to be very nearly Maxwellian [Langmuir, 1925; Gabor, Ash, Dracott, 1955] so that $f_{0-} \omega_{-}^{2}$ is invariant on the unperturbed orbits, which simplifies (14). Henceforth we consider only high frequencies,

$$
\omega \approx \omega_{-}>>\omega_{+},
$$

and neglect the ion contribution, $\mathscr{T}_{+}$. Then with the Maxwellian distribution

$$
f_{0-}=(\beta / \pi)^{3 / 2} e^{-\beta v^{2}},
$$

the conductivity kernel becomes

$$
\begin{aligned}
\mathscr{T}\left(\mathbf{x} ; \mathbf{x}^{\prime}\right)=-2 \omega^{2}(\mathbf{x}) \sum_{p} \int_{-\infty}^{\infty} \sigma_{p i} \\
\left(\boldsymbol{\nabla}-\frac{i \omega}{c^{2}} \mathbf{v}\right) \underline{e}_{i}^{-i \omega\left(t^{\prime}-t\right)} \beta f_{0-}(\mathbf{v}) \frac{\mathbf{v}^{\prime}}{v_{i}^{\prime}} d^{3} v,
\end{aligned}
$$

and in the electrostatic limit the kernel is

$$
\mathscr{S}\left(\mathbf{x} ; \mathbf{x}^{\prime}\right)=\frac{2 i \beta \omega_{-}^{2}(\mathbf{x})}{\omega} \sum_{p} \sigma_{p i} \int_{-\infty}^{\infty} \frac{\mathbf{v v}^{\prime}}{v_{i}^{\prime}} \cdot \underline{e}_{i}^{-i \omega\left(t^{\prime}-t\right)} f_{0-}(\mathbf{v}) d^{3} v .
$$

\subsection{General Method of Numerical Solution}

In the above form, (13) corresponds to a boundary value problem. The problem can be changed to a system of linear equations, which in principle can always be solved numerically. In the experiments the externally supplied driving field, $\mathbf{E}_{d}$, is always known. Split $\mathbf{E}$ into

$$
\mathbf{E}_{1}=\mathbf{E}_{d}+\mathbf{E}_{p},
$$

where $\mathbf{E}_{p}$ is due to sources in the plasma. Then (13) becomes

$$
\nabla^{2} \mathbf{E}_{p}+\frac{\omega^{2}}{c^{2}} \mathbf{E}_{p}=\int \mathscr{T}\left(\mathbf{x} ; \mathbf{x}^{\prime}\right)\left(\mathbf{E}_{p}\left(\mathbf{x}^{\prime}\right)+E_{d}\left(\mathbf{x}^{\prime}\right)\right) d^{3} x^{\prime},
$$

since $\mathbf{E}_{d}$ satisfies the free-space wave equation.

In matrix notation (19) has the form $A_{y}=b$, with solution $y=A^{-1} b$, where

$$
\begin{aligned}
y & =\text { column }\left(y_{1}, y_{2}, y_{3}\right), \\
y_{j} & =\operatorname{column}\left(E_{p j}\left(\mathbf{x}_{1}\right), E_{p j}\left(\mathbf{x}_{2}\right), \ldots\right),
\end{aligned}
$$

where $\mathbf{x}_{1}, \mathbf{x}_{2}, \ldots$ exhaust the space of $\mathbf{x}$. The inhomogeneous terms, $b$, is constructed from the driving term in (19) and has the same labeling arrangement as $y$. The matrix $A$ is a $3 \times 3$ supermatrix

$$
A=\left[A_{i j}\right],
$$

where the matrices $A_{i j}$ are, with $1, m$, being the labels given to points $\mathbf{x}_{1}, \mathbf{x}_{m}$, and $i, j$ being the labels on the components of $\mathbf{E}$,

$$
\left[A_{i j}\right]_{1 m}=\delta_{i j}\left(\frac{\omega^{2}}{c^{2}} \delta_{1 m}+L_{1 m}\right)-\left[\mathscr{T}_{i j}\right]_{1 m},
$$

where $L_{1 m} E_{j}$ is a difference representation of $\nabla^{2} E_{j}$.

In principle $A$ and $A^{-1}$ can be formed numerically for any system, e.g., cylindrical geometry. But in the cylinder problem (with no $z$-dependence), $\mathscr{T}$ has four components, each of which is a complex array formed by integration over two-dimensional orbits and is expensive in computer time. Inverting $A$ is also expensive, since it would necessarily be quite large. In the following two subsections we reduce the problem of the low-lying dipole modes of a thin cylindrical plasma to a one-dimensional problem, which we have solved numerically.

\subsection{Transit Resonance and the Coherence Length of an Orbit}

Drummond, Gerwin, and Springer [1961] pointed out that the orbit integral could have poles which arose from the resonances between the orbits and the fields. These transit poles arise whenever $\omega / 2 \pi$ is a harmonic of the transit frequency $1 / T$, where $T$ is the period of the particle orbit. For a slab of width $L$, with zero magnetic field and reflecting walls, $T=2 L / v$. In a magnetic field there are transit time poles at the cyclotron harmonics, and their effects have appeared in experiments [Bekefi, Coccoli, Hooper, and Buchsbaum, 1962]. However, they do not appear in the ionosphere backscatter experiments [Bowles, private communication]. The apparent reason has been given by Dougherty [1964], who solved a model FokkerPlanck equation, and found that the leading effect is not a simple collisional damping [Comisar, 1963], but rather a term which tends to destroy the poles. 
Consider a collisionless system in which all orbits are periodic. Go back to (12) and sum over successive periods of the orbit, which are identical except for a phase factor $e^{i p \omega T_{0}}$, where $T_{0}(\mathbf{x}, \mathbf{v})$ is the transit time on the collisionless orbit. Then the orbit integral becomes

$$
\begin{aligned}
& \int_{-\infty}^{t} \mathbf{E}_{1}\left(\mathbf{x}^{\prime}\right)-\boldsymbol{\nabla}_{v^{\prime}} f_{0 s}\left(\mathbf{v}^{\prime}\right) e^{-i \omega t^{\prime}} \omega_{s}^{2}\left(\mathbf{x}^{\prime}\right) d t^{\prime} \\
& =\left(\sum_{0}^{\infty} e^{i n \omega T_{0}}\right) \oint_{t-T_{0}}^{t} \mathbf{E}_{1}\left(\mathbf{x}^{\prime}\right) \cdot \boldsymbol{\nabla}_{v^{\prime}} f_{0 s} e^{-i \omega t^{\prime}} \omega_{s}^{2}\left(\mathbf{x}^{\prime}\right) d t^{\prime},
\end{aligned}
$$

where the integral includes one complete period of the orbit. The transit time poles arise from the sum

$$
\sum_{0}^{\infty} e^{i n \omega T_{0}}=1 /\left(1-e^{i \omega T_{0}}\right) .
$$

Since we have already adopted the Landau [1946] convention, we may assume an infinitesimal positive imaginary part of $\omega$ to force this sum to converge to (21). The transit time poles are at

$$
\omega_{m}=2 \pi m / T_{0} ; \quad m=0,1,2, \ldots .
$$

Note that the conductivity kernel equation becomes exact if we integrate along exact unperturbed orbits including collisions. The conductivity kernel equation then becomes equivalent to the single particle Liouville equation. Each orbit integral may be replaced by an average over many trials of the orbit. We assume it is sufficient to include only random, weak, coulomb collisions, so that the exact orbits are only slightly different from the collisionless orbits over times short compared to the relaxation time, $1 / \nu_{0}$. Then the magnitude of the left-hand side of (20) is less than

$$
\sum_{n=0}^{\infty}\left\langle e^{i n \omega T} n c\right\rangle I_{\max }(\mathbf{x}, \mathbf{v}),
$$

where $I_{\max }(\mathbf{x}, \mathbf{v})$ is the set of maximum values attained by the orbit integral over one period of the orbit, including points $x^{\prime}$ not reached on the collisionless orbit, and $T_{n c}$ is the transit time on the $n$th passage of a trial of the collisional orbit. We may reasonably expect that only the first term in the sum in (23) will be important if the $T_{n c}$ 's have a spread, $\triangle T$, satisfying

$$
\triangle T \geqslant 2 \pi / \omega \text {. }
$$

For $v$ on the order of $v_{\text {th }}$, and $\nu_{0} T<<1$, we have the approximate relations

$$
\frac{\triangle T}{T} \simeq \frac{\triangle v}{v}
$$

and

$$
\nabla v \simeq \nu_{0} T v
$$

which imply that

$$
\triangle T \simeq \nu_{0} T^{2}
$$

for weak collisions. Let $T \equiv T_{c}$ when the equality in (24) is satisfied. Then

$$
T_{c}=\left(\frac{2 \pi}{\omega \nu_{0}}\right)^{1 / 2} .
$$

If $\triangle T$ is the width of a Gaussian distribution of orbit times, we find that $\sum_{n=0}^{\infty}\left\langle e^{i n \omega T n c}\right\rangle=\sum_{0}^{\infty} e^{-\gamma n^{2}+i n \omega T_{0}}$, where $\gamma=\left(\omega \nu T_{0}^{2} / 2\right)^{2}$. When $T_{0}>T_{c}$ all but the first term in the sum are negligible, which supports (24).

For a plasma which is thick compared to a Debye length, $L_{D}, \triangle T$ may be much less than $T_{0}$ and still satisfy (24) for all electrons except those with such high energies that their number can be neglected. The condition for the existence of transit time poles is that the "coherence length" of the orbit must include many passages. This requirement is not satisfied by low-pressure mercury arcs with no or weak magnetic field or by the ionosphere [Dougherty, 1964]. With $L_{c}=\left(\pi \omega_{p} / \nu_{0}\right)^{1 / 2} L_{D}$ we find $L_{c}=10^{2} L_{D}$ for Dattner's data [1963], using standard estimates of the momentum changing frequency due to weak coulomb collisions, $\nu_{0}$.

However, we need not abandon the conductivity kernel formulation for the Fokker-Planck equation. The range $R$, of the kernel is short compared to typical orbit coherence lengths for almost any interesting plasma. To zeroth order in $R / L_{c}$ we may neglect collisions during the first passage from $x$ to $x^{\prime}$. Since the size of the plasma is many Debye lengths, $e^{i \omega T_{0}}$ is a rapidly oscillating function of $v$; the contribution to the kernel of any given passage after the first is very small. Even if $L_{c}$ is several times the plasma size the kernel will not be significantly affected by any but the first passage. We simply drop the higher passages on the orbit. Thus, while collisions nowhere appear explicitly in our conductivity kernel, they play a very important role in determining its form, and this is in spite of the fact that by the usual criterion, $\nu_{0}<<\omega$, collisions may be neglected.

\subsection{Reduction of the Cylinder Problem}

The highest noticeable resonance is generally observed to fall near the plasma frequency at the center of the discharge tube [Dattner, 1963; Schmitt, 1964], and the so-called "main" resonance, at roughly [Dattner, 1963] 0.4 of the highest plasma frequency (we have assumed that the average electron density is approximately one-half of the maximum). The lowest modes should be concentrated at relatively large radii, where $\omega \approx \omega_{p}(r)$. Their character is essentially one-dimensional for low-multipoles and long wavelengths in the $z$-direction, provided that transit time resonances are destroyed by fluctuations. 
Consider the scattering of plane waves by a thin, infinitely long, cylindrical plasma with $z$-symmetry. The driving field is

$$
\mathbf{E}_{d}=\hat{x} e^{i(k y-\omega t)},
$$

or

$\mathbf{E}_{d}=(\hat{r} \cos \theta+\hat{\theta} \sin \theta)[\cos (k r \sin \theta)+i \sin (k r \sin \theta)] e^{-i \omega t}$,

where $r, \theta$, are cylindrical polar coordinates and $x, y$ are rectangular coordinates in the $r, \theta$ plane, with $z$ along the cylinder axis and $\theta=0$ on the $x$-axis.

For a thin plasma of radius $a<<\lambda$, where $\lambda$ is the freespace wavelength, we have $k r \leqslant k a<<1$. In Dattner's experiments [1963] $k a$ ranges from 0.08 to 0.33 . Write

$$
\mathbf{E}_{d}=\hat{x} e^{-i \omega t}+\mathscr{O} k a,
$$

and neglect terms of order $k r$ for $r \leqslant a$, while retaining the full wave equation for $r>a$. This has been called the quasi-static approximation [Kaiser and Closs, 1952]. To zeroth order in $k a$ the conductivity kernel equation is $\left(13^{\prime}\right)$, where the kernel is given by $\left(17^{\prime}\right)$ without the sum over higher passages:

$$
\mathscr{S}\left(\mathbf{x} ; \mathbf{x}^{\prime}\right)=\frac{2 i \beta \omega_{-}^{2}(\mathbf{x})}{\omega} \int_{-\infty}^{\infty} \frac{\mathbf{v v}^{\prime}}{v_{i}^{\prime}} f_{0-}(\mathbf{v}) \underline{e}_{i}^{-i \omega\left(t^{\prime}-t\right)^{3}} d v
$$

From the symmetry of the unperturbed plasma the $\theta$-dependence of $\mathscr{S}$ at fixed $r$ must be of the form $g\left(\theta^{\prime}-\theta\right)$, which is sufficient to guarantee that driven oscillations have the same multipolarity as the driving field. Therefore, to zeroth order in $k a$ the fields are pure dipole modes,

$$
\mathbf{E}_{p}=\mathscr{E}(r) \hat{r} \cos \theta+\epsilon(r) \hat{\theta} \sin \theta,
$$

or

$$
\boldsymbol{\nabla} \cdot \mathbf{E}_{p}=\left(\mathscr{E}^{\prime}+\frac{\mathscr{E}}{r}+\frac{\epsilon}{r}\right) \cos \theta=-4 \pi e N_{1}(r) \cos \theta .
$$

To zeroth order in $k a$ [see the discussion above $\left(13^{\prime}\right)$ ]

$$
\boldsymbol{\nabla} \times \mathbf{E}_{p}=\frac{\sin \theta}{r}\left(\epsilon+r \epsilon^{\prime}-\mathscr{E}\right) \hat{z}=0 .
$$

Let $\lambda_{r}=\epsilon / \epsilon^{\prime}$ be the characteristic length and assume (as we have done everywhere in the static approximation, and as is verified by the results) that $\lambda_{r} / a<<1$. Then to lowest order in $k a$ and $\lambda_{r} / a$

$$
\epsilon^{\prime}=\mathscr{E} / r,
$$

and

$$
\epsilon=\mathscr{O}\left(\lambda_{r} / a\right) .
$$

Without transit time poles the terms of order $\lambda_{r} / a$ may be neglected when we substitute (26) into (13'), i.e., the off-diagonal kernel $S_{r_{\theta}}$ does not contribute to the equation for $\mathscr{E}$ because $\epsilon$ is small.
Since the range, $R$, of the kernel in any direction should be only a few Debye lengths, the $\theta^{\prime}$ dependence may be expanded,

$$
\cos \theta^{\prime}=\cos \theta+\mathbb{O}(R / a)^{2} .
$$

Neglecting the term $\mathscr{O}(R / a)^{2}$, the $\cos \theta$ dependence can be cancelled out of the conductivity kernel equation for all $\theta$ such that $\cos \theta>>k a$.

By choosing $x_{i}=r$ we can now do the $\theta^{\prime}$ and $z^{\prime}$ integrations immediately [see the definition of $\underline{e}_{i}^{-i \omega\left(t^{\prime}-t\right)}$ (10)], and obtain the one-dimensional equation

$$
\mathscr{E}(r)=\int_{0}^{a} \mathscr{K}\left(r ; r^{\prime}\right)\left(\mathscr{E}\left(r^{\prime}\right)+E_{d}\left(r^{\prime}\right)\right) d r^{\prime},
$$

where

$$
\mathscr{K}\left(r ; r^{\prime}\right)=\frac{2 i \beta \omega_{-}^{2}(r)}{\omega} \int_{-\infty}^{\infty} v f_{0-}(v) \underline{e}^{-i \omega\left(t^{\prime}-t\right)} d v,
$$

where $\underline{e}^{-i \omega\left(t^{\prime}-t\right)}$ is just a step function at the turning point multiplied by $e^{-i \omega\left(t^{\prime}-t\right)}$. The orbit times, $t^{\prime}-t$, are now calculated in a one-dimensional potential, the corrections being $\mathscr{O}(R / a)$.

For a given energy there are two terms in (28), one connecting $r$ and $r^{\prime}$ directly, the other connecting them through a reflection from the nearest turning point. The kernel is symmetric.

For $r \geqslant a$ we have

$$
\nabla^{2} \mathbf{E}_{p}+\frac{\omega^{2}}{c^{2}} \mathbf{E}_{p}=0
$$

The appropriate solution of (29) must be chosen by requiring continuity of $\mathbf{E}_{p}$ at $r=a$. Therefore a measure of the scattered radiation is

$$
\epsilon(a)=\int_{r_{0}}^{a} \frac{\mathscr{E}(r)}{a-r_{0}} d r+\epsilon\left(r_{0}\right) \frac{r_{0}}{a-r_{0}} .
$$

If the radiation does not penetrate significantly into the plasma beyond $r_{0}$, then

$$
\boldsymbol{\epsilon}(a)=\frac{1}{\theta} \int_{r_{0}}^{a} \mathscr{E}(r) d r
$$

so that to zeroth order in $\frac{\epsilon\left(r_{0}\right)}{\epsilon(a)}$ the radiation fields are proportional to the "dipole moment,"

$$
D=\int_{r_{0}}^{a} \mathscr{E}(r) d r .
$$

The outgoing wave solution of (29) for the $\theta$-component of $E_{p}$ is.

$$
\epsilon(r)=A H_{1}^{(1)}(k r),
$$

where $k=\omega / c$ and $H_{1}^{(1)}$ is the Hankel function of type 1 . The boundary condition is

$$
\epsilon(a)=D / a .
$$


The average power radiated away per unit length is

$$
P_{\text {rad }}=\lim _{r \rightarrow \infty}\left(r \epsilon^{2}(r)\right) / 4 .
$$

Define the incident power as

$$
P_{\text {inc }}=2 a E_{d}^{2} / 4 \pi,
$$

and define the dipole scattering width, $d$, by

$$
d=a P_{\text {rad }} P_{\text {inc. }}
$$

The dipole scattering width is one half of Herlofson's isotropic scattering width. For small $k a$ the first term in the asymptotic series for $H_{1}^{(1)}$ gives $A$, and the scattering width is given by

$$
\frac{d}{a}=\frac{\pi}{4}(k a)^{2} \frac{D D^{*}}{a^{2}}
$$

The approximations we have made to reduce the cylinder problem to a one-dimensional problem correspond to the following model, in which it is assumed that the $y, z$ dependence of $E_{d}$ can be neglected: The slab of plasma is bounded by a flat inner wall at $x=0$ and a flat insulating wall at $x=w$. The inner wall emits a half-Maxwellian distribution of electrons and absorbs all electrons which strike it. A sheath is built up on the insulating wall. The infinitely massive ions are distributed in such a way as to satisfy Poisson's equation. This model could be duplicated very closely if the inner wall were a hot tungsten plate emitting cesium plasma.

It is convenient to scale (27) and (28) by measuring distances in Debye length times $\sqrt{2}$ and time in plasma periods. Let $x=0$ be an arbitrary position, and let

$$
L_{D}=\left(2 k T / m \omega_{p}^{2}(0)\right)^{1 / 2} .
$$

The scaling is

$$
\begin{aligned}
x & \rightarrow x L_{D}, \\
t-t^{\prime} & \rightarrow \tau / \omega_{p}(0), \\
\varphi_{0} & \rightarrow-k T \varphi_{0} / e, \\
E & \rightarrow-k T E / L_{D} e, \\
\omega & \rightarrow \omega_{p}(0) \Omega, \\
\beta v^{2} & \rightarrow w,
\end{aligned}
$$

where $\varphi_{0}(0)=0$. We will usually pick $x=0$ at the inner wall. The scaled longitudinal kernel becomes

$$
K\left(x, x^{\prime}\right)=\frac{e^{-\varphi_{0}(x)}}{i \Omega \sqrt{\pi}} \int_{0}^{\infty} e^{-w} \sum_{ \pm} \underline{e}^{i \Omega \pi\left(x, w, \pm, x^{\prime}\right)} d w,
$$

where \pm is the sign of $v$. Note that, since $\varphi_{0}(x)+w=w_{0}$ is invariant on the orbit, the kernel is symmetric.
The insulating wall which confines the positive column is not included in the conductivity kernel as written above. Unperturbed orbits with energy above the floating potential do not return to the plasma, which is accounted for by not including orbits after they have hit the wall. The oscillating electric fields will cause some electrons to reach the wall even though their unperturbed orbits do not, and vice versa. This causes a perturbation in the number of reflected orbits and therefore in the current and the electric field. Let $v_{c}(x)$ be the velocity of an electron whose unperturbed orbit reaches the wall with $v_{c}^{\prime}=0$, i.e.,

$$
v_{c}^{\prime}\left(x, v_{c} ; x_{w}\right)=0 .
$$

To first order in the perturbation $\delta v_{c}$ of $v_{c}$, the perturbed current at $(x, t)$ due to perturbations in the wall current is

$$
\delta j=-e \beta N_{0}(x)\left(\frac{\pi}{\beta}\right)^{1 / 2} e^{-\beta v_{c}^{2}(x)} v_{c}(x) \delta v_{c}(x, t) .
$$

Integrating along the unperturbed orbit

$$
\begin{aligned}
\delta v_{c}=-\frac{e}{m} \int_{-\infty}^{t-t^{\prime}\left(x, v_{c}, x_{w}\right)} & E_{1}\left(x^{\prime}\right) e^{j \omega\left(t-t^{\prime}\right)} d t^{\prime} \\
& =-\frac{e}{m} e^{i \omega t^{\prime}\left(x, v_{c}, x_{w}\right)} \int_{0}^{\infty} E_{1}\left(x^{\prime}\right) e^{i \omega T} d T,
\end{aligned}
$$

where the orbit starts at the wall. Because we drop all transit time resonances $\delta v_{c}$ is nonsingular. Therefore

$$
\delta j=\mathscr{O} e^{-\beta v_{c}^{2}},
$$

which may be neglected if we consider only $\omega$ 's such that the oscillations are concentrated in regions where $\exp \left(-\beta v_{c}^{2}(x)\right)<<1$. In the plasma exp $\left(-\beta v_{c}^{2}\right)$ $=\mathscr{O}\left(m / m_{+}\right)^{1 / 2}$.

A similar one-dimensional problem-driven oscillations of the sheath on a semi-infinite uniform plasma-has been solved numerically by Pavkovich [1963] for frequencies $\omega<\omega_{p}(s)$. The properties of this system and the non-uniform plasma with a sheath are very different.

\subsection{Numerical Solution of the Conductivity Kernel Equation}

The numerical solution of the conductivity kernel equation was performed on the computers of the University of California at San Diego and the National Center for Atmospheric Research. One program formed the conductivity kernels and stored them on magnetic tapes. A second program read the kernels from the tapes, called a standard subroutine which inverted the kernels by the Gauss-Jordan method, and formed the solution, $E_{p}$.

Efficiency of the inversion program required that the matrices needed for the inversion be stored en- 
tirely in memory, which limited us to about 70 points on the $x$-axis. Actually, 61 points were used, and this was very satisfactory. For several reasons efficiency decreased especially rapidly for errors in the kernel of less than a few tenths of one percent, so errors were held to that level. The resulting errors in $E_{p}$ range from less than one percent to a few percent. The calculation required one hour of machine time for a batch of 20 values of $\Omega$.

All integrations were done as simple sums. When the path length, $x-x^{\prime}$, was long, the phase, $\Omega \tau\left(x, v ; x^{\prime}\right)$ sometimes varied by even more than $2 \pi$ between neighboring values of $v$ on the integration mesh, so that even though the mechanics of the integration were unchanged (simple sum) those portions of the velocity integration were actually done by sampling [Meyer, 1954]. Accuracy of the integration was checked both by examining the convergence of the kernel and the fields as the step sizes were made smaller, and by comparison with the Landau kernel [Pavkovich and Kino, 1963; Pavkovich, 1963] in the case of very weak fields.

\subsection{Results From the Conductivity Kernel Method}

Experimental data [Gabor, Ash, and Dracott, 1955; Gierke, Ott, and Schwirzke, 1961; Harp and Kino, 1963] on are structure was used as a guide, but because of considerable uncertainty in the plasma frequency at the sheath edge it was necessary to try various values of the steady fields. Since the field measurements and plasma resonance experiments have not been carried out on the same plasmas, there was no point in searching for steady fields which would give $D(\Omega)$ closely resembling, say, Dattner's curves.

All of the systems studied had a constant field, $E_{0}$, in the plasma, smoothly joined to a harmonic oscillator field, $\left(x-x_{0}\right) \Omega_{s h}^{2}$, in the sheath, where $x_{0}<s$ is such that $E_{0}=\left(s-x_{0}\right) \Omega_{s h}^{2}$. We give results for two systems which differ in $E_{0}, \Omega_{s h}$, and thickness. With $E_{0}$ and $\Omega_{s h}$ scaled to the sheath edge, system 1 has $E_{0}=0.121, \Omega_{s h}=0.746$, which is a reasonable fit to Gabor, Ash, and Dracott [1955] data. System 2 has stronger fields, $E_{0}=0.232$ and $\Omega_{s h}=1.93$. The relative sheath strength, $\Omega_{s h}^{2} / E_{0}$, is about three times greater than in system 1 . System 1 is relatively thicker, i.e., over the full range of $\Omega$ the total oscillating field has insignificant penetration to the inner wall; but system 2 is damped by the inner wall if $\Omega>0.40$.

The most important characteristic of the oscillations excited in the plasma is the magnitude of the dipole moment,

$$
D_{m}=\left(D D^{*}\right)^{1 / 2}
$$

The energy stored in the macroscopic variables at any time is equal to the amplitude of the field energy,

$$
\mathscr{E}=\frac{1}{8 \pi} \int_{0}^{x_{w}} E_{p} E_{p}^{*} d x
$$

The rate of dissipation of energy must balance the power absorbed from the external field; i.e.,

$$
P_{\mathrm{diss}}=-\frac{\Omega}{8 \pi} \int_{0}^{x_{w}} E_{d} \operatorname{Im} E_{p} d x
$$

or

$$
P_{\text {diss }}=-\Omega E_{d} \operatorname{Im} D / 8 \pi,
$$

for uniform $E_{d}$. The number of cycles required to dissipate the macroscopic energy, $\mathscr{E}$, would be

$$
Q=\mathscr{E} /\left(2 \pi E_{d} \operatorname{Im} D\right),
$$

and the phase shift of the scattered radiation is $\theta=\tan ^{-1}$ $(\operatorname{Im} D / \operatorname{Re} D)$. Figures $2 \mathrm{a}$ and $2 \mathrm{~b}$ are plots of $\mathscr{E}, D_{m}$, $\operatorname{Im} D, Q, \theta$ versus $\Omega$ for systems 1 and 2 , respectively. The frequencies are scaled to the plasma frequency at the inner wall. Both systems show peaks in the dipole moment.

System $l$ is the more interesting. The dipole moment and energy have two strong resonances and at least two more weak peaks. The strong resonances occur at nearly the same frequencies for both $\mathscr{E}$ and $D$.

Calculating the scattering width at the first resonance from (38) we find

$$
d / \lambda=8.5(k a)^{2},
$$

where we have set $a$ equal to the thickness of our slab of plasma. At $k a=0.3$, which is certainly near the upper limit for the quasi-static approximation, $d / a=15$, or $d / \lambda=0.7$. For $k a=0.1, d / a=5$ and $d / \lambda=0.08$, and even $k a=0.05$ gives $d / a=2.7, d / \lambda=0.02$. A cylindrical plasma corresponding to system 1 would certainly have $d / a>1$, and might come within a factor of two of Herlofson's cold plasma result, $d / \lambda=4 / \pi$, which was obtained as the radiation damping limit.

The relative damping, $\frac{1}{Q}$, is uncorrelated with $\mathscr{E}$,

but the coupling to the driving field, $D$, has sharp peaks at the resonances. In this sense plasma resonance is due to maxima in the coupling rather than the other possibility, minima in the damping [Leavens, 1963].

Figure 3 shows $E_{p}$ for several values of $\Omega$. Beyond the second node of $E_{p}$ the extrema are very weak, because of Landau damping. The damping may even be large for the first half-cycle of $\boldsymbol{E}_{p}$ if it falls near the sheath edge, because the electrons reflected from the sheath tend to be out of phase with the unreflected electrons $\left[\Omega_{\mathrm{sh}} \approx 0.75 \Omega_{p}(s)\right]$. This is apparent in the kernels, three rows of one of which we show in figure 4. The two rows with the diagonal far from $s$ are similar except for a scale factor. 

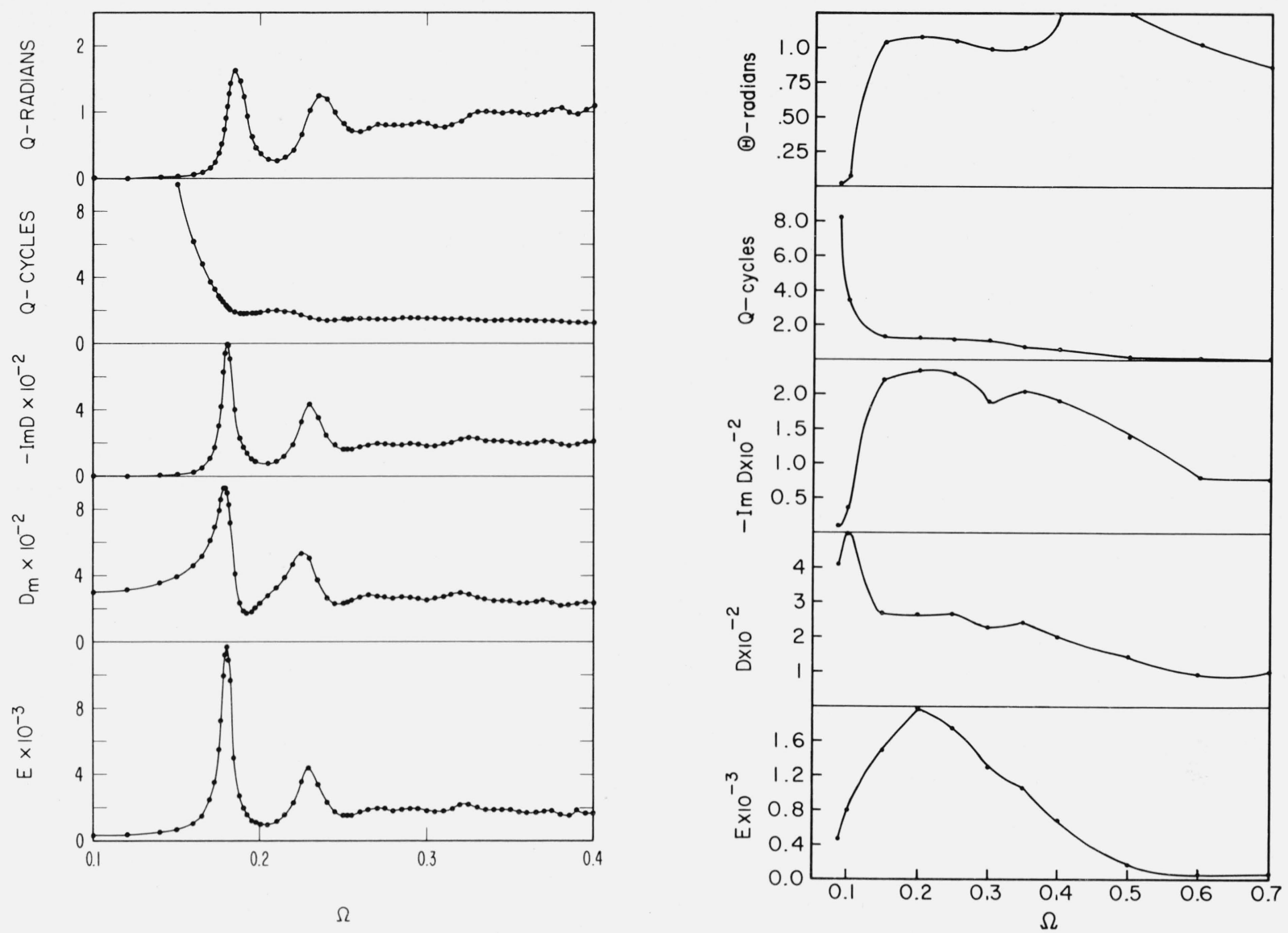

Figure 2. Macroscopic energy, dipole moment, imaginary part of the dipole moment, quality, and phase shift versus $\Omega$ for: (a) System 1; (b) System 2. Scaled to plasma frequency and Debye length at $\mathrm{x}=0$.

Driving field $=1$.

System 2 has one strong resonance and two weak resonances. Above $\Omega=0.40$ the field penetrates to the inner wall, which apparently causes enough damping to prevent any more peaks from occurring. Multiplying the frequencies in system 2 by two will scale them to the same base as those in system 1 . The positions and spacings of the peaks are quite different from system 1. The $Q(\Omega)$ curve has even less relationship to the $D(\Omega)$ curve. The first resonance is weaker than in system 1 , but could still have $d / a>1$ for reasonable values of $a$ and $k$.

We have studied several other systems with stronger steady fields. One of them was a very thin system, so that the total $E=E_{p}+E_{d}$ penetrated to the inner wall. All showed well defined peaks in $D(\Omega)$, but none had the strong resonances of system 1 . The effect of a stronger field on the electron orbits is to decrease $t\left(x, v ; x^{\prime}\right)$, lowering the minimum velocity for which $\omega t<<2 \pi$, and thereby enhancing the imaginary part of $K$ near the diagonal and weakening the real part, so more damping is to be expected with stronger fields.

We would like to emphasize the following:
Any relationship between the first resonance frequency and the average plasma frequency is purely an accidental consequence of the form of the unperturbed plasma. This is clear because the portions of the plasma into which the fields do not penetrate can be modified freely without affecting the calculation. This implies that the partial success of cold plasma theories [Tonks, 1931a, b; Herlofson, 1951; Crawford, 1963] in describing the first resonance is also accidental. There is no difference of kind between the first and higher resonances. It is especially worth emphasizing that all of the resonances come from a single model, and one should not add an extra cold plasma resonance to the resonances found from kinetic theory or fluid theory [Crawford, 1963]. There is nothing fundamental about the series limit [Dattner, 1963; Crawford, 1963]. The higher resonances produce relatively weak changes in the dipole moment because of Landau damping. Furthermore, the Landau damping can be expected to increase when $\Omega$ is greater than the largest plasma frequency. These two effects presumably bring resonances above the "series limit" down to an undetectably small amplitude. 

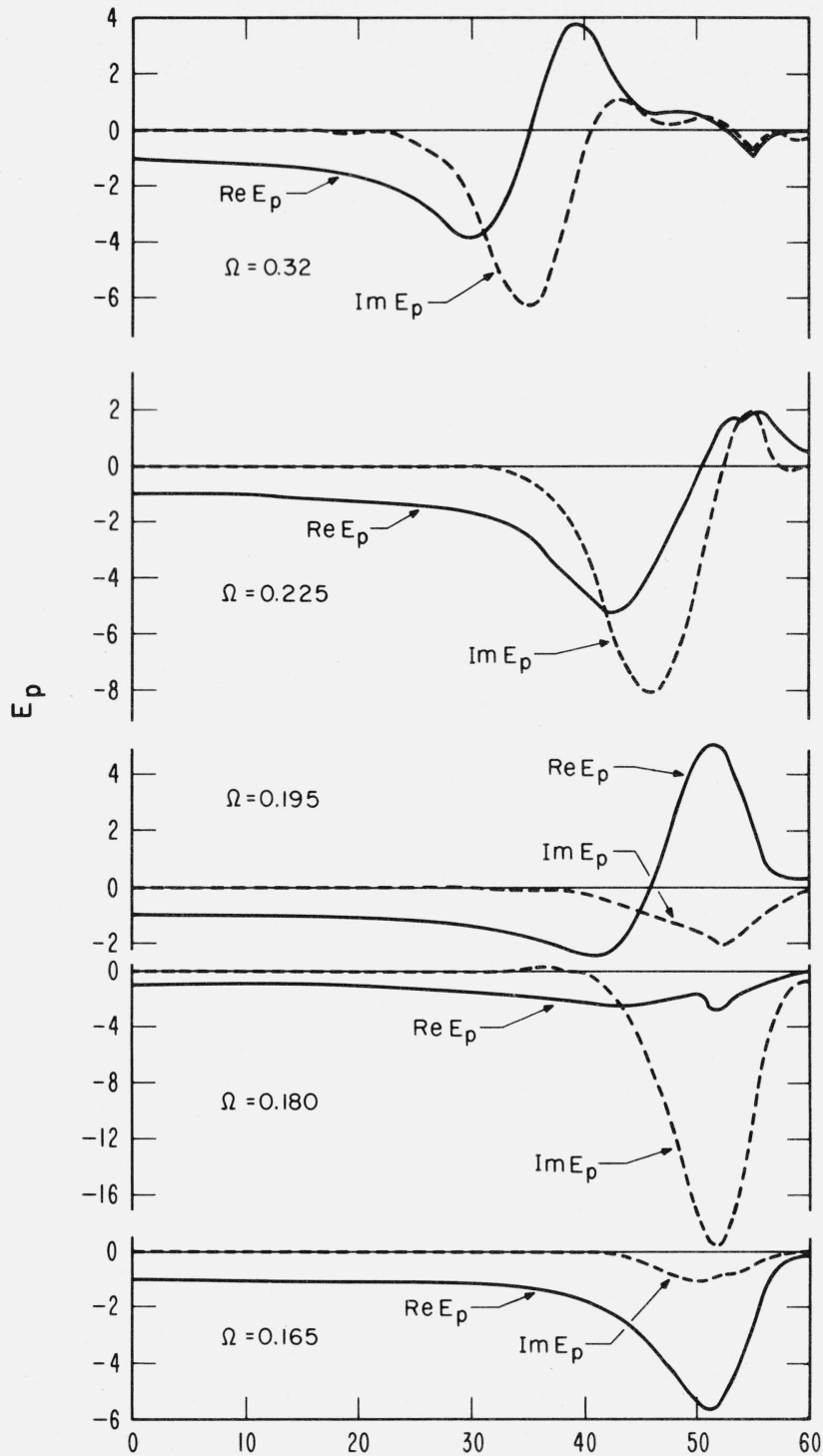

$I_{X}$

FIGURE 3. Real and imaginary parts of $\mathrm{E}_{\mathrm{p}}$ versus position. System 1.

\section{Adiabatic Fluid Theory}

In this section we, (1) sketch the derivation of a linearized fluid-type equation of motion by the usual procedure of taking moments of the electron Vlasov equation and terminating the set by making the adiabatic approximation; (2) consider possible "boundary conditions" on the fluid equation; and (3) calculate resonance spectra.

\subsection{Fluid Equations for Electrostatic Oscillations}

The first two moments of the Vlasov equation are

$$
\frac{\partial N}{\partial t}+\boldsymbol{\nabla} \cdot(N<\mathbf{v}>)=0,
$$

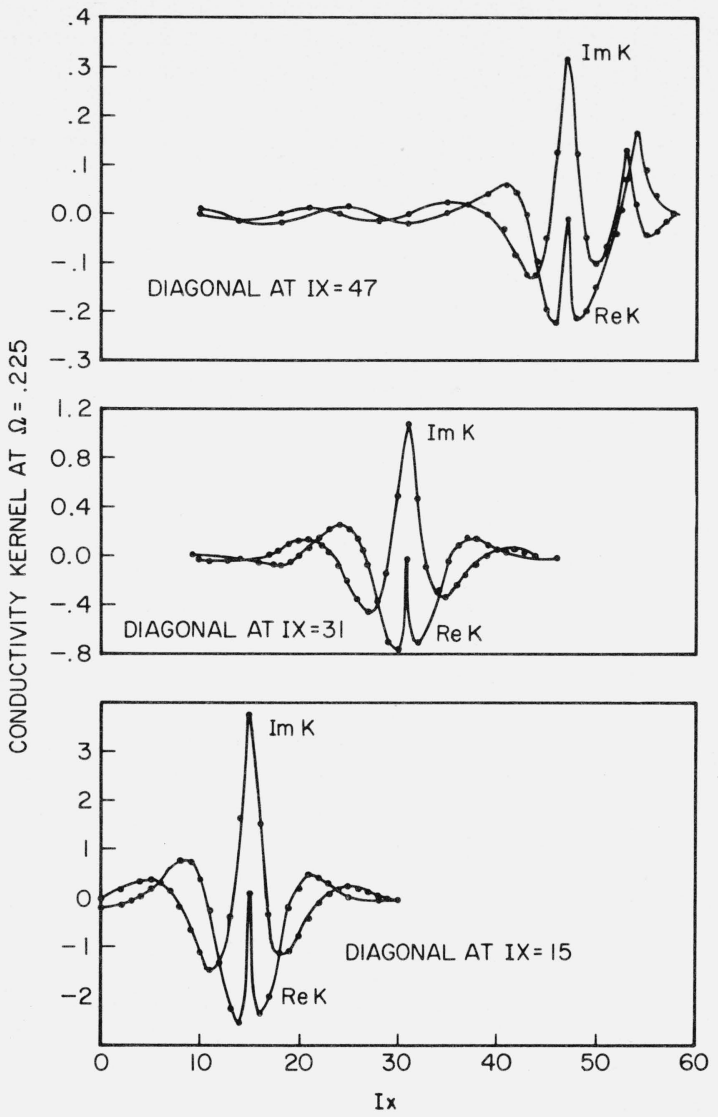

FiguRE 4. Three rows of the conductivity kernel. $\Omega=.255$. System 1 .

$$
\frac{\partial(N<\mathbf{v}>)}{\partial t}+\boldsymbol{\nabla}\left(N<v^{2}>\right)+\frac{N e \mathbf{E}}{m}=0,
$$

where $N$ is the electron density, and

$$
<v^{n}>=\int_{-\infty}^{\infty} v^{n} f(v) d v
$$

In what follows we use $\mathbf{v}=\langle\mathbf{v}\rangle+\mathbf{u},<\mathbf{u}>=0$,

$$
<v_{x}^{2}>=<v_{x}^{2}>+<u_{x}^{2}>, u^{2}=3<u_{x}^{2}>\text {. }
$$

The set of moment equations is terminated by taking the second moment equation and setting $\left\langle u_{i} u_{j} u_{k}\right\rangle$ $=0$. The same approximation is made more easily

by the adiabatic approximation $\left(\frac{\partial}{\partial t}+<\mathbf{v}>\cdot \nabla\right)$ $\left(P N^{-\gamma}\right)=0$, with $\gamma=3$ since only one degree of freedom is involved. Linearizing the moment equations and using Poisson's equation we find the equation of 
motion for one-dimensional problems,

$$
E_{p}^{\prime \prime}+\frac{3 e E_{0}}{m u^{2}} E_{p}^{\prime}+\frac{1}{u^{2}}\left(\omega^{2}-\omega_{p}^{2}(x)+\frac{2 e E_{0}^{\prime}}{m}\right) E_{p}=\frac{\omega_{p}^{2}}{u^{2}} E_{d} .
$$

The normal form of (54) is

$$
y^{\prime \prime}+\left(q^{2}-p^{2}\right) y=-\frac{\omega_{p}}{u^{2}} E_{d},
$$

where

$$
q^{2}=\omega^{2} / u^{2}, p^{2}=\left[\omega_{p}^{2}+\frac{e E_{0}^{\prime}}{2 m}-\frac{9}{2 u^{2}}\left(\frac{e E_{0}}{m}\right)^{2}\right] / u^{2},
$$

and

$$
E_{p}=\omega_{p}(x) y
$$

To derive (54) from the moment equations, terms in $v_{0}, \nabla v_{0}$, and $\nabla u^{2}$ are neglected. For the electrons this is justified to within a few $k T$ (potential drop) of the insulating wall. The fluid equation scales with the Debye length. The same scaling used in section 2 gives

$$
E_{p}^{\prime \prime}+E_{0} E_{p}^{\prime}+\frac{2}{3}\left(\Omega^{2}-\Omega_{p}^{2}+E_{0}^{\prime}\right) E_{p}=\frac{2}{3} \Omega_{p}^{2} E_{d},
$$

or

$$
y^{\prime \prime}+\frac{2}{3}\left[\Omega^{2}-\Omega_{p}^{2}+\frac{E_{0}^{\prime}}{4}-\frac{3}{8} E_{0}^{2}\right] y=\frac{2}{3} \Omega_{p} E_{d} .
$$

Different fluid equations are sometimes obtained by the isothermal approximation or by making the adiabatic approximation in the laboratory reference frame. The differences can be important. For example, if $\varphi_{0}$ is a harmonic oscillator potential the requirement that $E_{p}$ be zero at infinity gives a continuous spectrum of free $\left(E_{d}=0\right)$ oscillations for (54), but a discrete spectrum for the similar equation obtained by making the adiabatic approximation in the laboratory frame [Weibel, 1960]. The latter result disagrees with results obtained from the Vlasov equation [Weibel, 1960]. It is well known that the adiabatic fluid equations can give reasonable values for the splitting of the resonance frequencies [Gould, 1960; Leavens and Abramoff, 1961; Fejer, 1962; Crawford, 1963; Leavens, 1963; Nickel, Parker, and Gould, 1963; Weissglas, 1963; Hoh, 1964]. The conductivity kernel solutions suggest a "resonance condition" which we apply to the fluid equations in the next section.

\subsection{Resonance Condition}

There is no reason to impose boundary conditions on the plasma oscillations, and indeed the kinetic theory results indicated a continuous spectrum.
It may be, however, that we can find approximate conditions for resonance which would be used in the same way as boundary conditions. The conductivity kernel solutions suggest that we might be able to predict resonance frequencies with the fluid equations by using the $E_{1}(s)=0$ boundary condition in the problem of free oscillations, but that only the odd eigenvalues are resonances. For slowly varying amplitude (as a function of $\Omega$ ) this corresponds roughly to maximum dipole moment. The resonance splitting is twice that given by a rigid wall boundary condition at the sheath edge.

\subsection{Resonance Spectrum}

In the domain $0 \leqslant x \leqslant s$, the coefficient $p^{2}$ in $\left(56^{\prime}\right)$ is relatively slowly varying and for weak fields the $E_{0}^{2}$ and $E_{0}^{\prime}$ terms may be neglected, only the variation of $\Omega_{p}$ being significant. The free oscillation equation is

$$
y^{\prime \prime}+\frac{2}{3}\left(\Omega^{2}-\Omega_{p}^{2}(x)\right) y=0,
$$

where

$$
E_{p}=\Omega_{p} y .
$$

The asymptotic solution of (57) which becomes exponentially small into the plasma is [Heading, 1962]

$$
E_{p}=\frac{\Omega_{p}(x)}{g^{1 / 2}(x)}\left(\int_{x_{0}}^{x} g d x\right)^{1 / 6} A i\left[-\left(\frac{3}{2} \int_{x_{0}}^{x} g d x\right)^{2 / 3}\right]
$$

where

$$
g^{2}(x)=\frac{2}{3}\left(\Omega^{2}-\Omega_{p}^{2}(x)\right), \quad g^{2}\left(x_{0}\right)=0,
$$

and $A i(\xi)$ is the Airy function. The only zeroes of $E_{p}$

\begin{tabular}{|c|c|c|c|c|}
\hline$i=1$ & 3 & 5 & 7 & 9 \\
\hline$\xi_{i}=2.34$ & 5.52 & 7.94 & 10.04 & 11.94 \\
\hline$\Omega_{i}=0.178$ & 0.239 & 0.286 & 0.324 & 0.365 \\
\hline
\end{tabular}
are in $A i(\xi)$. Let $A i\left(-\xi_{i}\right)=0$, then the condition for resonance is

$$
\left(\frac{3}{2} \int_{x_{0}}^{x} g(x) d x\right)^{2 / 3}=\xi_{i}, \quad i=1,3,5, \ldots
$$

For constant $E_{0}$ this reduces to

$\frac{E_{0}}{\sqrt{6}} \xi_{i}^{3 / 2}=-\Omega_{i}\left(\nu_{i}-\tanh ^{-1} \nu_{i}\right) ; \quad \nu_{i}=\left(1-e_{0}^{i E_{0} s} / \Omega_{i}^{2}\right)^{1 / 2}$.

Solving (60) graphically for system 1, we find the following frequencies for the first five resonances:

The $\xi_{i}$ were taken from the British Association tables of the Airy function [Miller, 1946]. The $\Omega_{i}$ are scaled to the plasma frequency at $x=0$. The agreement 
between the $\Omega_{i}$ and the positions of the peaks on the $D(\Omega)$ curve for system 1 is quite good; $\Omega_{1}$ is in error by 1 percent, and the average spacing is extremely close. For system 2, we find

$$
\begin{array}{lllll}
\Omega_{i}=0.103 & 0.156 & 0.194 & 0.228 & 0.259 .
\end{array}
$$

The first resonance agrees extremely well with the conductivity kernel solution, but the splitting is a factor of two too small.

It is interesting to compare the fluid solutions for $E_{p}$ (fig. 5) and the kinetic solutions, figure 3. The plotted fluid solutions were actually obtained by numerical integration of (56), but they agree with (58). Direct comparison is impossible, one set of solutions being for the driven problem, the other for free oscillations. Nevertheless, it is clear that the fluid solutions are not very satisfactory. The fluid equations are useless well into the sheath, where their solutions exponentiate extremely rapidly [Leavens, 1963]. Otherwise, the main qualitative difference is the more rapid decrease of the amplitude of successive extrema as one moves toward the wall in the case of the conductivity kernel solutions. The success in calculating the resonance frequencies merely reflects the fact that the spatial nodes of the fluid solutions have approximately the correct spacing.

\section{Summary and Conclusions}

Plasma resonance in nonuniform plasmas bounded by sheaths has been studied by the conductivity kernel method. Although this is a collisionless method we were able to account for the dominant effect of weak collisions by cutting off orbit integrations when the path length became greater than the coherence length, i.e., when an ensemble of electrons released from the point $(x, v)$ would reach $x^{\prime}$ after times which would have a spread of more than $2 \pi / \omega$. The coherence length is much shorter than the mean free path; for Dattner's arcs it is approximately one plasma diameter. With the orbit cutoff it was possible to reduce the problem of low lying dipole resonances of a cylinder to a one-dimensional problem.

The one-dimensional problem of steady state driven oscillations was solved numerically for a model which used a linear potential in the plasma, continuously joined to a harmonic oscillator potential in the sheath. The model has plasma resonances. The most interesting static potential (which was also probably the best fit to experimental measurements of the static fields near the sheath) had two strong resonances and at least two weaker ones.

The program to solve the conductivity kernel equation will work for any static potential, but as yet resonance experiments and static field measurements in the sheath and the plasma have not been done on the same arc. Cesium plasmas would be ideal for such an experiment. With a hot, flat, tungsten plate facing an insulating plate across a gap of $\sim 50$ Debye lengths
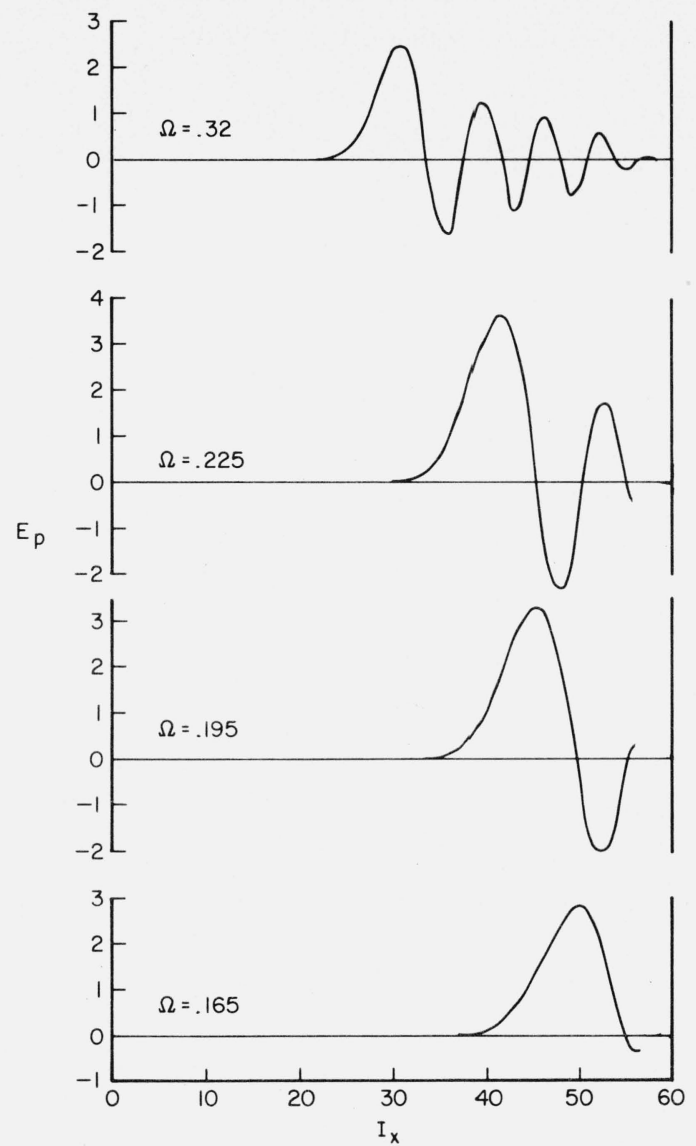

FiguRE 5. Fluid solutions of the free oscillation problem, $\mathrm{E}_{\mathrm{p}}$ versus position.

System 1.

the experiment would correspond exactly to the onedimensional problem solved by our program, and the field measurements would not be too difficult.

Although Landau damping of the conductivity kernel solutions was important, we found that the adiabatic fluid equations, with a "boundary condition" chosen to correspond approximately to maximum coupling to the driving field, gave an extremely accurate prediction of the resonance frequencies in the most realistic case studied, and predicted the first resonance quite accurately (i.e., agreeing with the conductivity kernel solutions) even for a system with overly strong static fields. Qualitative comparison with Dattner's data [1963] shows that the relative spacing of our calculated resonances is too large for the higher resonances, which is expected because we have used a model which has an increasing density gradient into the plasma. The "level spacing" is, however, in qualitative agreement with Dattner's data.

In conclusion, we note that the "boundary condition" was inferred from the conductivity kernel solutions in the neighborhood of the sheath. The sheath not only influences the line shapes through Landau damping, but also has a strong influence on the positions of the resonances. 
It is a pleasure to thank M. N. Rosenbluth for his guidance. We are grateful for the cooperation of the computer centers of the University of California at San Diego and the National Center for Atmospheric Research. This research was supported by the U.S.A.E.C., the University of California, and the National Bureau of Standards.

\section{References}

Bekefi, G., J. D. Coccoli, E. B. Hooper, Jr., and S. J. Buchsbaum (1962), Microwave emission and absorption at cyclotron harmonics of a warm plasma, Phys. Rev. Letters 9, No. 1, 6-9.

Billam, E. R., and I. C. Browne (1955), Meteors, ed. T. R. Kaiser (Pergamon Press, Ltd., London and New York, N.Y.).

Boley, F. I. (1958), Scattering of microwave radiation by a plasma column, Nature 182, 790-791.

Comisar, G. G. (1963), Collisional damping of plasma oscillations, Phys. Fluids 6, No. 1, 76-82.

Crawford, F. W. (1963), Resonances of a cylindrical plasma column, Microwave Laboratory Report No. 1045, W. W. Hanson Lab. Stanford University.

Dattner, A. (1957). The plasma resonator, Ericsson Technics 13 , No. 2, 309-350.

Dattner, A. (15 Mar. 1963), Resonance densities in a cylindrical plasma column, Phys. Rev. Letters 10, No. 6, 205-2-6.

Dougherty, J. P. (1964), Model Fokker-Planck equation for a plasma and its solution, Phys. Fluids 7, No. 11, 1788-1799.

Drummond, J. E., R. A. Gerwin, and G. B. Springer (1961), The concept of conductivity, J. Nucl. Energy, Part C: Plasma Phys. 2, 98-108.

Fejer, J. A. (1962), Scattering of electromagnetic waves by a plasma cylinder, General Motors Report No. TR62-209L.

Gabor, D., E. A. Ash, and D. Dracott (1955), Langmuir's paradox, Nature 1 76, 916-919.

Gierke, G. V., W. Ott, and F. Schwirzke (1961), Fifth International Conference on Ionization Phenomena in Gases, Munich.

Gould, R. W. (1960), Scattering from a plasma column, California Institute of Technology Report No. 1, Contract DA36-039 SC85317.

Harp, R. S., and G. S. Kino (1963), Measurement of fields in the plasma sheath by an electron beam probing technique, Microwave Laboratory Report No. 1076, Stanford University.

Heading, J. (1962), An Introduction to Phase-Integral Methods, p. 29 (Methuen and Co., Ltd., London).

Herlofson, N. (1951), Plasma resonance in ionosphere irregularities, Arkiv Fysik 3, 247-297.

Hoh, F. C. (1964), Longitudinal oscillations in a nonuniform plasma, Phys. Rev. 133, No. 4A, A1016-A1020.

Kaiser, T. R., and R. L. Closs, (1952), Theory of radio reflections from Meteor trails. I, Phil. Mag. 43, 1-32.
Landau, L. D. (1946), On the vibrations of the electronic plasma, J. Physics USSR 10, 25-34.

Langmuir, I. (1925), Scattering of electrons in ionized gases, Phys. Rev. 26, 585-613.

Leavens, W. M. (1963), Effect of the sheath on plasma resonance, Meeting of the Division of Plasma Physics, American Society, San Diego, Calif.

Leavens, W. M., and R. Abramoff (1961), Theory of plasma resonance, Convair Report ZPH-093.

Lustig, C. D. (1964), Microwave noise resonances from a plasma column, Phys. Letters 9, No. 4, 315-317.

Meyer, H. A. Editor (1954), Symposium on Monte-Carlo Methods (John Wiley and Sons, Inc., New York, N.Y.).

Miller, J. C. P. (1946), British Association Tables, Part-Volume B.

Nickel, J. C., J. B. Parker, and R. W. Gould (1963), Resonance oscillations in a hot nonuniform plasma column, Phys. Rev. Letters 11, $183-185$.

Ott, W. (1963), Ein Versuch zur Klärung des Langmuir-Paradoxons, report of the Institute für Plasmaphysik, Munchen.

Panofsky, W. K. H., and M. Phillips (1962), Classical Electricity and Magnetism, 2d ed. ch. 11 (Addison-Wesley Publishing Co., Inc., Reading, Mass.).

Parker, J. V.. J. C. Nickel, and R. W. Gould (1964), Resonance oscillations in a hot nonuniform plasma, Phys. Fluids 7, No. 9, 14891500 .

Pavkovich, J. (1963), Numerical calculations related to the R.F. properties of the plasma sheath, Meeting of the Division of Plasma Physics, American Physical Society, San Diego, Calif.

Pavkovich, J., and G. S. Kino (1963), R. F. behavior of the plasma sheath, Meeting of the Division of Plasma Physics, American Physical Society, San Diego, Calif.

Romell, D. (1951), Radio reflexions from a column of ionized gas, Nature 167, 243.

Schmitt, H. J. (1964), Acoustic resonances in afterglow plasma, Appl. Phys. Letters 4, No. 6, 111-112.

Self, S. A. (1963), Exact solution of the low-pressure plasma sheath equation, Microwave Laboratory Report No. 1009, Stanford University.

Thompson, W. B. (1962), An Introduction to Plasma Physics, p. 182 (Pergamon Press, London).

Tonks, L. (1 June 1931a), The high frequency behavior of a plasma, Phys. Rev. 37, 1458-1483.

Tonks, L. (15 Sept. 1931b), Plasma electron resonance, plasma resonance and plasma shape, Phys. Rev. 38, 1219-1223.

Weibel, E. S. (1960), Oscillations of a nonuniform plasma, Phys. Fluids 3, No. 3, 399-407.

Weissglas, P. (1963), Resonance oscillations in a hot nonuniform plasma, Phys. Rev. Letters 10, No. 6, 206-209.

Wolff, P. A. (1956), Theory of plasma resonance, Phys. Rev. 103, No. 4, 845-850.

(Paper 69D10-566) 